\title{
MULTISENSOR IMAGE FUSION TECHNIQUES IN REMOTE SENSING
}

\author{
Manfred Ehlers \\ Surveying Engineering Program \\ University of Maine \\ Orono, ME 04469, USA \\ Commission VII
}

\begin{abstract}
Current and future remote sensing programs such as Landsat, SPOT, MOS, ERS, JERS, and the space platform's Earth Observing System (Eos) are based on a variety of imaging sensors that will provide timely and repetitive multisensor earth observation data on a global scale. Visible, infrared and microwave images of high spatial and spectral resolution will eventually be available for all parts of the earth. It is essential that efficient processing techniques be developed to cope with the large multisensor data volumes. This paper discusses data fusion techniques that have proved successful for synergistic merging of SPOT HRV, Landsat TM and SIR-B images. Examples are given for integrative rectification, enhancement of cartographic feature extraction and improvement of spatial resolution.
\end{abstract}

\section{INTRODUCTION}

Earth observing systems of the future such as the proposed polar orbiting space platforms of NASA, ESA and Japan will likely bring another dimension to remote sensing. A variety of imaging (and non-imaging) sensors will be employed to cover the full range of the electromagnetic spectrum available for remote sensing of the earth (Butler et al., 1986). For example, a $30-\mathrm{m}$ resolution imaging spectrometer will provide image data with a spectral coverage of 0.4 to $2.5 \mu \mathrm{m}$ and a spectral resolution of 9.4 to $11.7 \mathrm{~nm}$ (Goetz et al., 1987). This amounts to 196 simultaneously recorded spectral bands. In addition, other sensors will provide information in different spectral bands (e.g., thermal infrared and microwave) and/or at different spatial resolutions yielding data volumes and spectral band combinations for which efficient processing methods are yet to be developed.

This multisensor, multispectral, multiresolution, multitemporal information will eventually be available for all parts of the earth and presents a data processing challenge to the remote sensing society that has to be addressed. Integrative processing techniques have to fuse the multiimage information to make it useful for a user community that is concerned with mapping, monitoring and modeling the earth's components (figure 1). This paper presents image fusion methods and algorithms that proved successful for synergistic processing of SPOT High Resolution Visible (HRV), 
Landsat Thematic Mapper (TM) and Shuttle Imaging Radar (SIR-B) data.

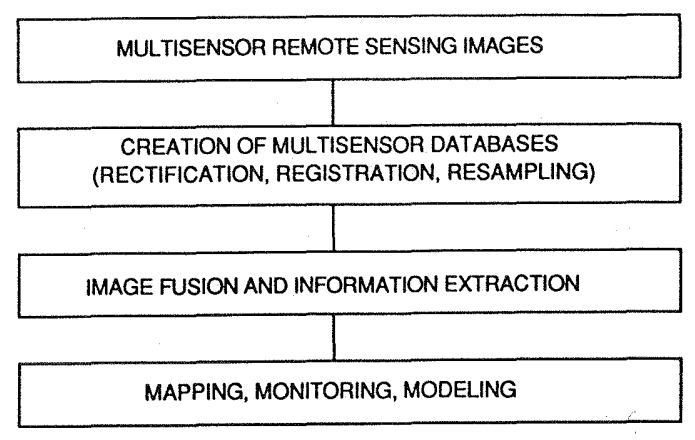

Figure_1. Concept for multisensor image evaluation.

\section{INTEGRATIVE RECTIFICATION}

Earth-related integrative processing of multisensor image data requires that all images are in register to each other and georeferenced to a common ground coordinate system (figure 2). Rectification and image registration are well known and documented techniques in remote sensing (Bernstein et al., 1983; Welch, Jordan and Ehlers, 1985). Since an image-to-image registration approach is easier to accomplish and allows the utilization of automated or semi-automated image processing techniques it may prove necessary to rectify only one image of the multisensor dataset to ground control. Other multisensor or multitemporal image data of the same area may be registered to the rectified reference image using automatic or visual techniques (Ehlers, 1984; Luhmann and Ehlers, 1984; Welch, 1984).

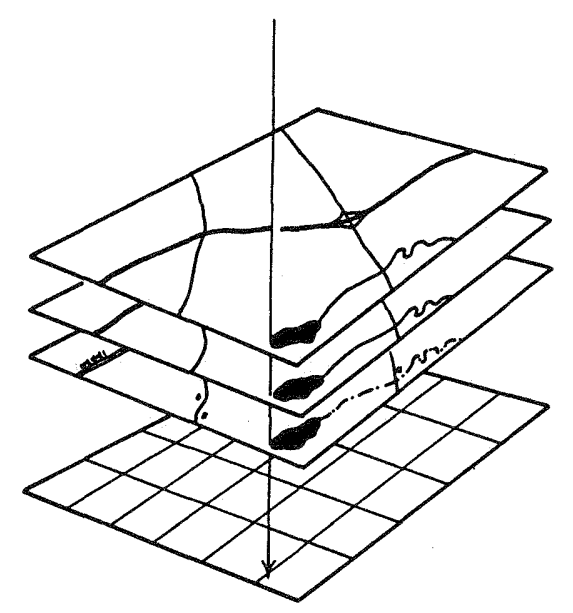

Elqure 2. Concept of an image database referenced to ground control.

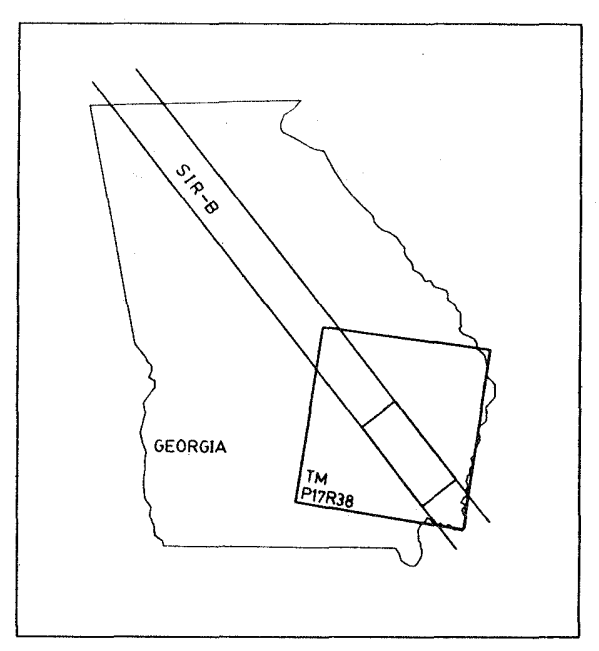

Eiqure 3. TM and SIR-B image data of southeast Georgia. 
The validity of this integrative rectification approach could be demonstrated for Landsat TM and SIR-B data. A study area common to satellite scenes of both sensors was identified in the Southeast of the United States (figure 3). This study area encompasses $90 \times 90 \mathrm{~km}$ and is equivalent to the southeast quadrant (quad 4) of the TM scene. Using a least squares based first degree polynomial rectification algorithm, the TM quad was rectified to the Universal Transverse Mercator (UTM) coordinate system yielding a root-mean-square error $\left(R M S E_{X Y}\right)$ of $\pm 11.1 \mathrm{~m}( \pm 0.4 \mathrm{TM}$ pixel) at eleven withheld check points. By contrast, RMSE $X Y$ values for the rectified SIR-B dataset were about $\pm 27.5 \mathrm{~m}$ to $\pm 30.9 \mathrm{~m}( \pm 2.2$ to \pm 2.5 SIR-B pixels) at the withheld check points and the ground control points (GCPs), respectively.

The major difference in the two datasets is the 'quality' of the images which in return determines the accuracy to which control points in the images can be defined. With TM image data, GCPs can be located to a fraction of a pixel whereas for SIR-B data even well defined GCPs such as road intersections cannot be determined to better than \pm 1 to \pm 3 pixels. To overcome these obstacles, an iterative-integrative rectification procedure for low resolution and low signal-to-noise ratio (SNR) data (such as synthetic aperture radar (SAR) or thermal infrared) was developed (Ehlers, 1987). As initial step, the low resolution image is coarsely registered to the rectified reference image of high resolution. Only a few relative control points (tie points) are necessary to accomplish this task. Once the two images are in approximate register, additional tie points can be identified by displaying the images in a flicker mode on the screen of an image processing system. These additional tie points may then be used for a precise registration, and the procedure can be repeated until a sufficient accuracy has been achieved (figure 4 ).

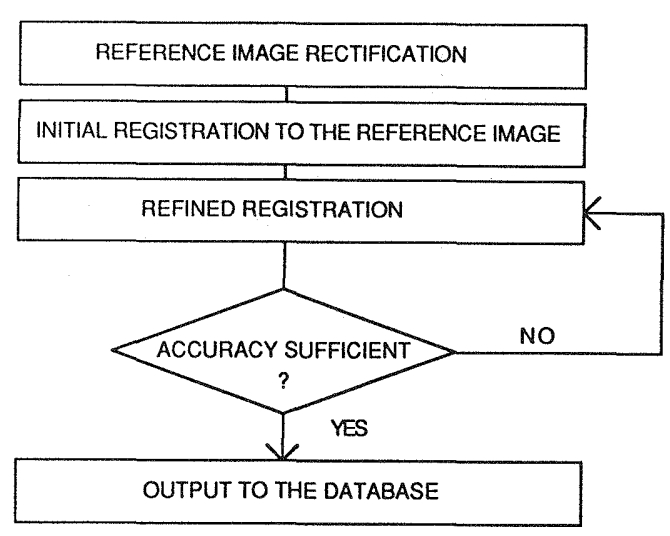

Figure 4. Integrative rectification procedure.

The results for the integrative rectification approach are illustrated in figure 5 . 


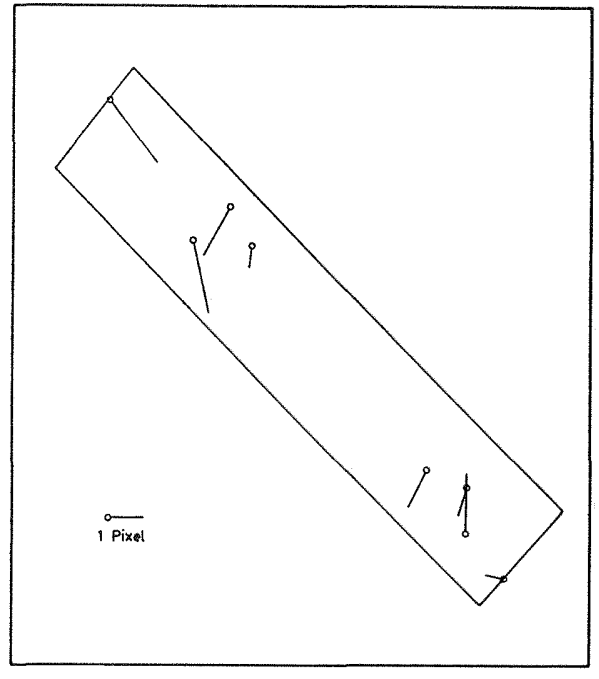

(a)

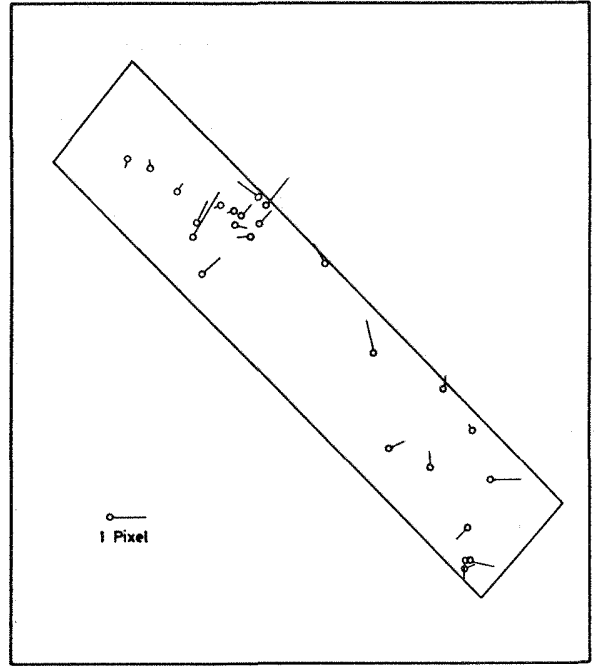

(b)

Eigure 5. (a) Error vectors at 8 check points for the initial registration of SIR-B to TM; (b) Error vectors at 25 check points for the final registration.

The initial registration error of $\pm 1.37 \mathrm{TM}$ data pixels $( \pm 39.0 \mathrm{~m}$ ) (figure $5 \mathrm{a}$ ) was reduced to \pm 0.57 pixel $( \pm 16.2 \mathrm{~m}$ ) in the second step (figure $5 \mathrm{~b}$ ) and could not be improved in a third iteration. Overall, the RMSE $X Y$ value of the integrated rectification approach $\left\{R M S E_{X Y}\right.$ (int)\} can statistically be described as a combination of two independent stochastic variables: (a) the error associated with the rectification of the reference image to the ground coordinate system $\left\{\operatorname{RMSE}_{X Y}(\right.$ ref)\} and (b) the image-to-image registration error $\left\{\mathrm{RMSE}_{X Y}(\mathrm{reg})\right\}$. Thus, the integrated rectification accuracy may be estimated as follows:

$$
\operatorname{RMSE}_{X Y}(\text { int })= \pm \sqrt{\left\{\mathrm{RMSE}^{2} X Y(\text { ref })+\operatorname{RMSE}^{2} X Y(\text { reg })\right\}}
$$

With RMSE $X Y$ values of $\pm 11.1 \mathrm{~m}$ for the TM reference image and $\pm 16.2 \mathrm{~m}$ for the registration of SIR-B to TM, the accuarcy of the integrative rectification approach according to equation (1) is about $\pm 19.6 \mathrm{~m}$ which represents a significant improvement over the $\pm 30.9 \mathrm{~m}$ obtained in the previous rectification. Similar results with this methods have been reported for other TM/SIR-B datasets (Anderson, 1987).

\section{SYNERGISTIC FEATURE EXTRACTION}

To maximize information content for visual interpretation from multisensor datasets, it may be necessary to modify the traditional red-green-blue (RGB) approach, i.e., assign individual images or spectral bands to the RGB guns of the image processing display (figure 6a). Commonly employed alternatives include: (a) adding a single band or a single image to all other image layers (Chavez, 1986); (b) applying 


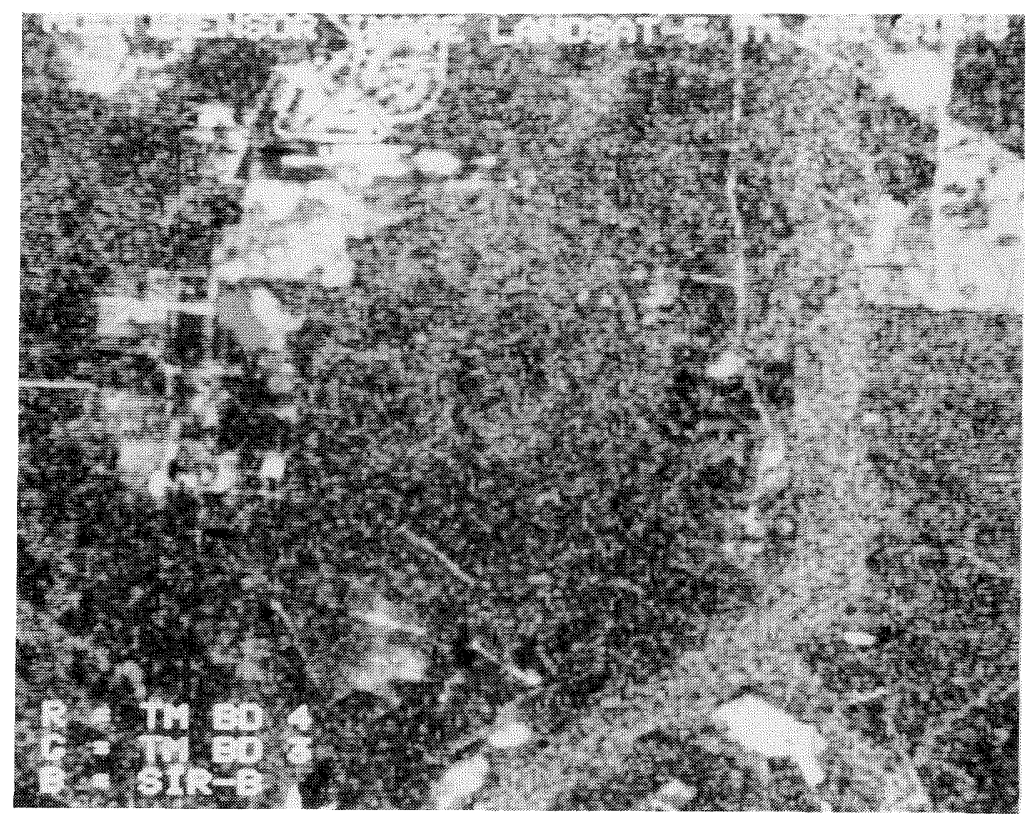

(a)

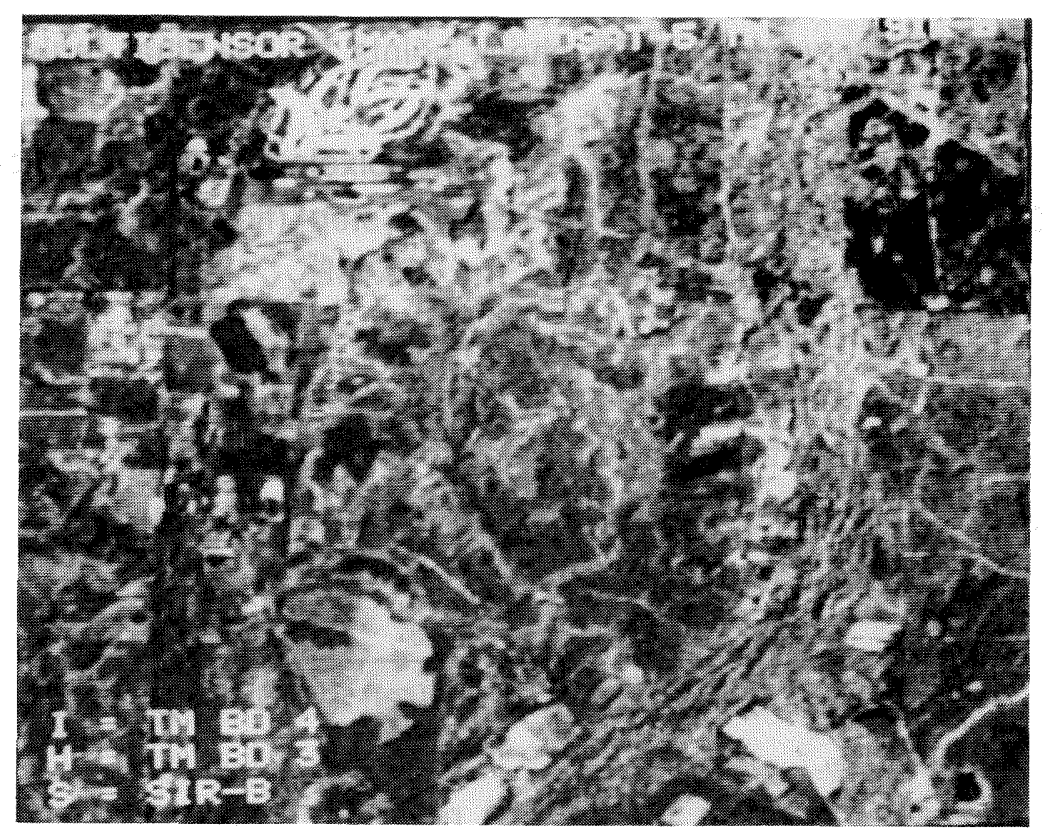

(b)

Figure 6. (a) Black and white print of an RGB TM/SIR-B false color composite with TM bands 4 (red) and 3 (green) and SIR-B (blue). The high speckle noise of the SIR-B data makes the location of cartographic features extremely difficult. (b) Black and white print of an IHS display of the merged TM/SIR-B dataset with TM bands 4 (intensity) and 3 (hue) and SIR-B (saturation). The IHS display provides better discrimination and is relatively noise free. 
principal component or decorrelation stretch transforms to the image layers (Niblack, 1986); and (c) make use of the intensity-hue-saturation (IHS) color transform (Haydn et al., 1982). Of these approaches, the IHS transform appears most useful for efficient integration of very dissimilar images (Koger, 1984). Noisy or low resolution image layers such as SAR or thermal infrared images, can be assigned to the saturation component, whereas high resolution/high SNR data can modulate the intensity and hue components, respectively (figure $6 \mathrm{~b}$ ).

To assess the merits of the multisensor TM/SIR-B dataset, cartographic features were digitized from single image and IHS enhanced image layers and compared to corresponding features on U.S. Geological Survey (USGS) 1:24,000; 1:100,000; and $1: 250,000$ scale topographic maps. Features shown on these maps were grouped into linear, areal and point features, and then manually digitized to establish reference values for completeness by category at each map scale. The ratio of feature information extracted from the satellite data to the reference values for the maps determined the percent completeness values (Welch and Ehlers, 1988).

Approximately 40 to 70 percent of the planimetric information depicted on maps of $1: 24,000$ to $1: 250,000$ scale could be extracted from the SIR-B data, whereas 55 to 85 percent completeness values could be obtained from the TM image. These values, however, could be increased to about 65 to 95 percent completeness for the IHS enhanced TM/SIR-B multisensor dataset (figure 7). The potential for using IHS fused TM/SIR-B images for map compilation and revision even at a scale of 1:24,000 is illustrated in figure 8.

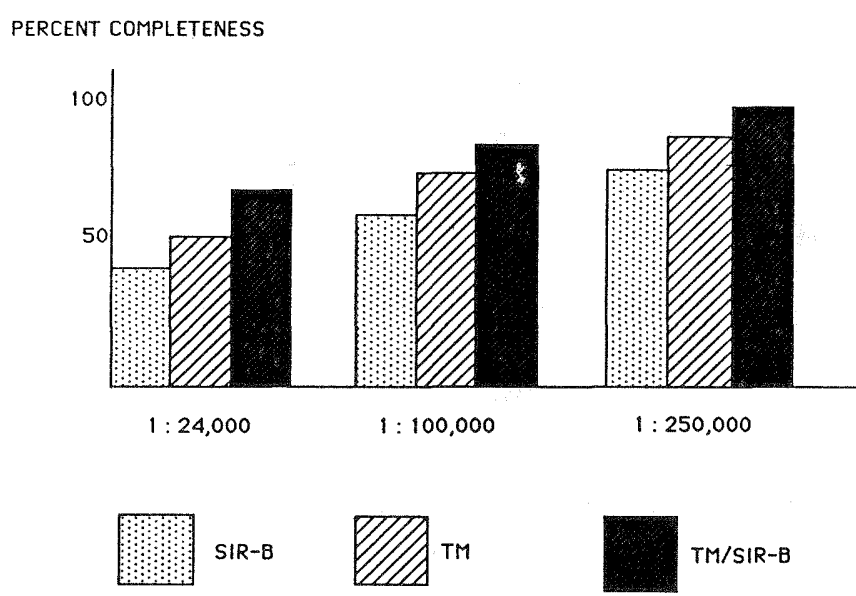

Eiqure 7. Percent completeness of map detail as a function of map scale for the TM, SIR-B and IHS merged TM/SIR-B datasets.

\section{IMAGE SHARPENING}

A very direct approach to image enhancement is the use of high resolution data, e.g. SPOT HRV 10-m panchromatic images, to sharpen images of lower spatial resolution, e.g. TM or SPOT HRV multispectral data. Once a set of multisensor images is 
placed in register with a high resolution reference image, the digital numbers (DNs) of the various multispectral bands may be merged with those for the single band (panchromatic) reference image using techniques previously described by Cliche et al. (1985) or Chavez (1986). These methods may be summarized in the following equations:

$$
\begin{aligned}
& D N_{i}{ }^{\prime}=\alpha_{i}^{*} \sqrt{D N_{i}^{*} D N(h)}+\beta_{i} \\
& D N_{i}=\alpha_{i}^{*}\left(\gamma_{i}{ }^{*} D N_{i}+\delta_{i}^{*} D N(h)\right)+\beta_{i}
\end{aligned}
$$

where $D N_{i}$ and $D N_{i}$ are the $D N s$ for the ith band of the original and fused multispectral image, respectively; $D N(h)$ is the $D N$ for the high resolution reference image; $\alpha_{i}, \beta_{i}$ are scaling factors; and $\gamma_{i}, \delta_{i}$ are weighting factors.

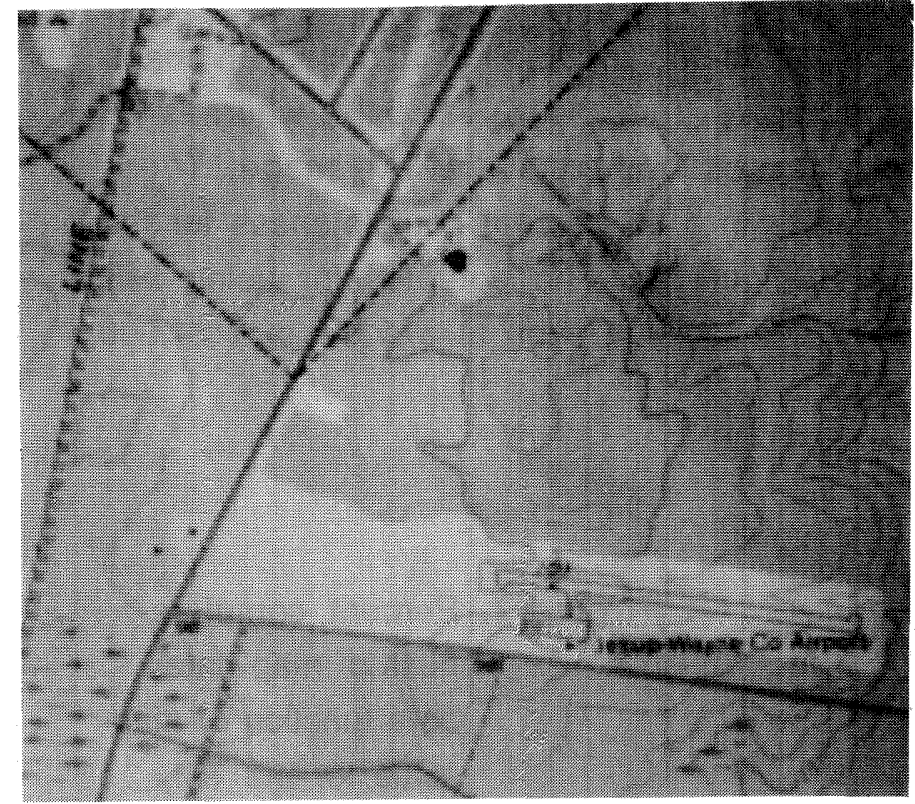

(a)

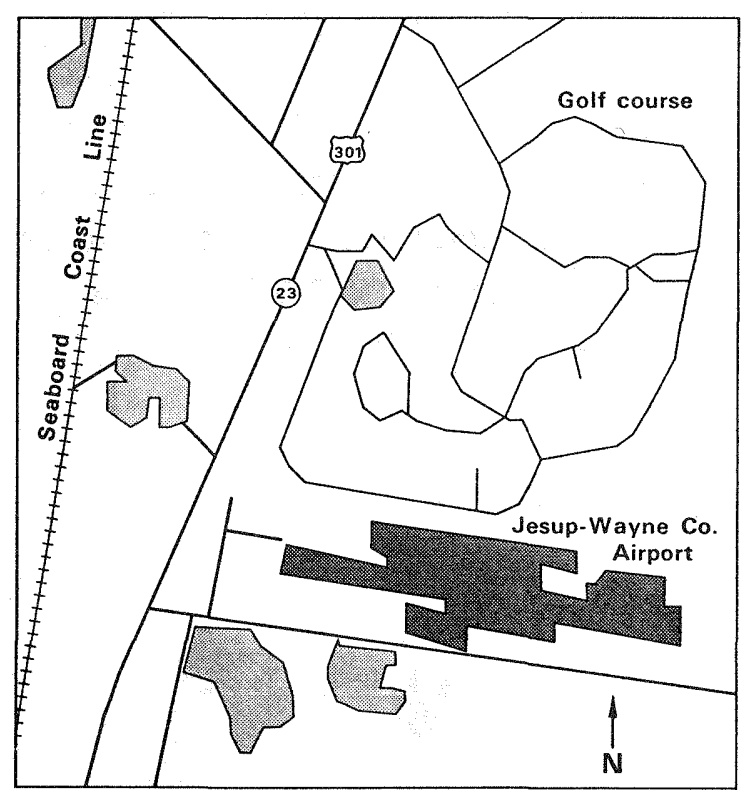

(b)

Figure 8. (a) Video-digitized subset of the 1:24,000 scale reference map and (b) planimetric map subset compiled from the IHS merged TM/SIR-B dataset revealing significant temporal changes to the map compiled in 1970.

A disadvantage of these algorithms is that the sharpened multispectral image bands have a higher spectral correlation than the original bands. Thus, the improvement in spatial resolution is accomplished with a loss in spectral information. However, images of superior spectral discrimination and improved spatial resolution 
can be achieved using the IHS color transform (Welch and Ehlers, 1987). Three selected spectral bands from $28.5-\mathrm{m}$ TM or $20-\mathrm{m}$ SPOT HRV multispectral data that can be displayed as RGB on the image processing display are transformed into the IHS domain. The DNs of 10-m SPOT panchromatic data (or other high resolution images) are then substituted for the intensity component and transformed back into the RGB domain (figure 9).

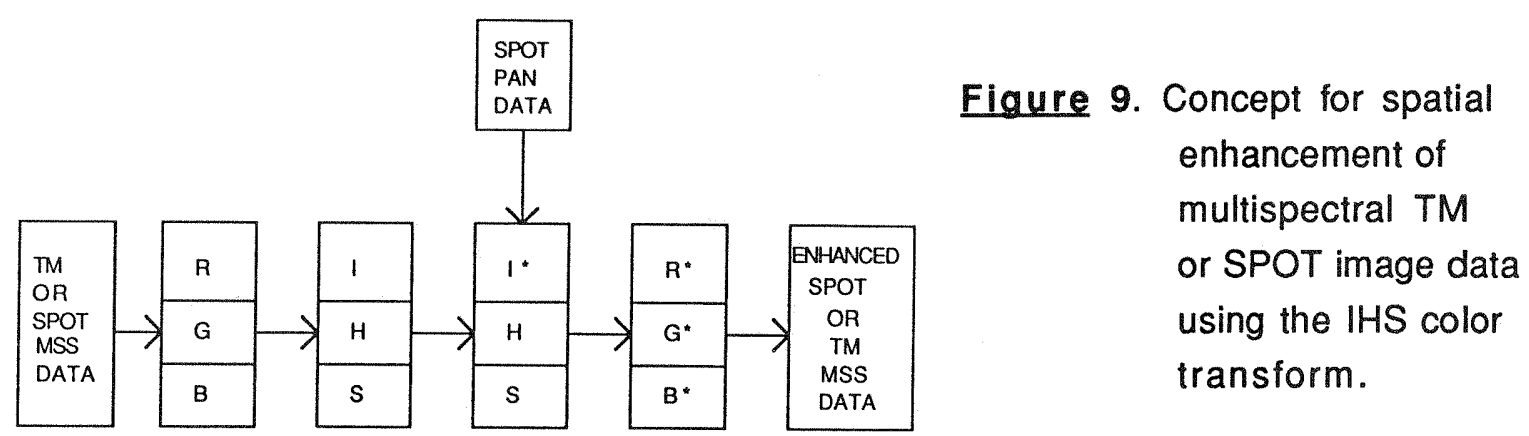

The resulting multisensor and multiresolution image retains the spatial resolution of the 10-m SPOT reference image, yet provides the spectral characteristics (hue and saturation values) of the TM or SPOT multispectral data. Interestingly, the amount of detail in spatially enhanced TM images is absolutely comparable to that in enhanced SPOT products (figure 10). Consequently, SPOT panchromatic images may be used to enhance existing TM datasets and create multisensor, multiresolution, multitemporal image products of improved interpretability and quality.

\section{CONCLUSION}

Planimetric accuracy for SIR-B data could be improved from $\pm 30 \mathrm{~m}$ RMSE $_{X Y}$ to $\pm 20 \mathrm{~m}$ RMSE $X Y$ using an integrative rectification/registration approach by merging SIR-B with TM image data of superior quality and resolution. Using IHS color transform techniques, significantly more cartographic information could be obtained from merged TM/SIR-B composites than from single SIR-B or TM images. IHS enhanced TM/SIR-B composite images improved the map information content about 10 to 25 percent over TM and SIR-B images, respectively.

Striking enhancements in the quality of SPOT and TM multispectral images of 28.5- $\mathrm{m}$ and $20-\mathrm{m}$ resolution can be realized by using the IHS transform to merge the multispectral bands with SPOT $10-\mathrm{m}$ panchromatic data. The resulting multispectral composites have spatial resolution properties similar to the panchromatic SPOT image, yet retain the spectral discrimination qualities of the original dataset.

Overall, significant improvements in rectification accuracy, detail of cartographic 
features and interpretability can be realized using multisensor image fusion techniques. Enhanced multisensor data products will prove useful to scientists seeking to maximize the amount of information that can be extracted from satellite image data.

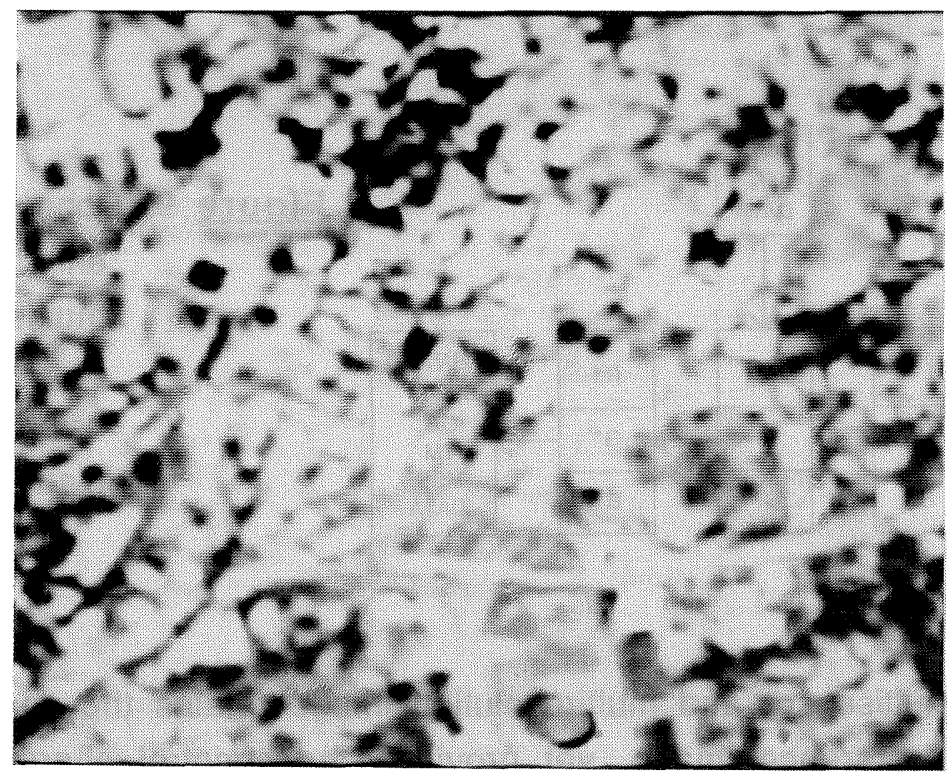

(a)

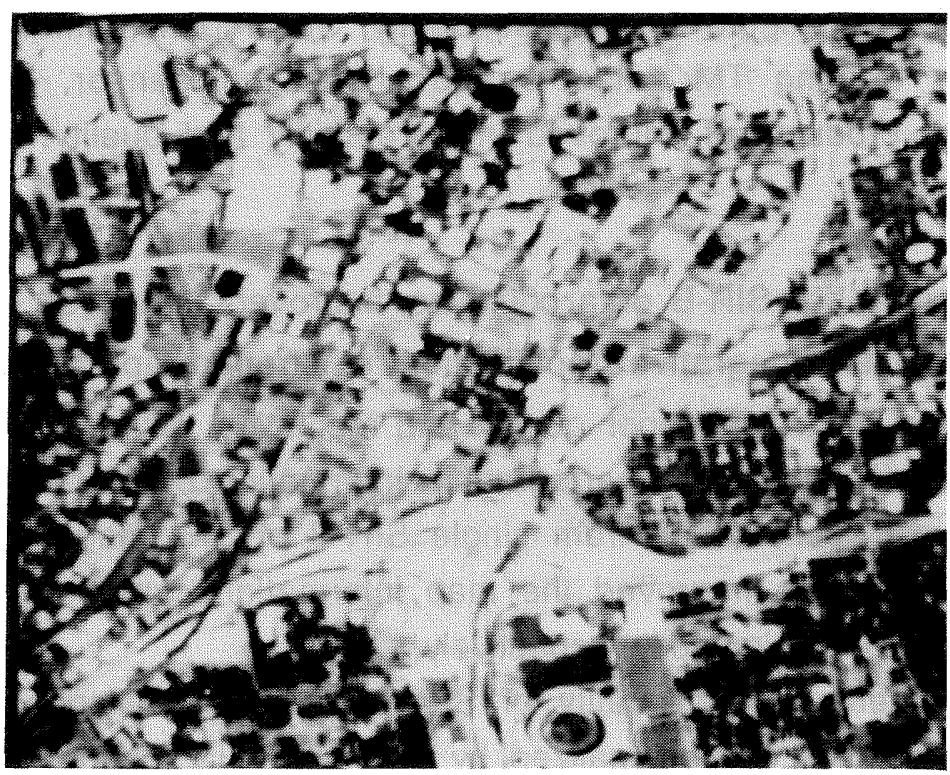

(b) 


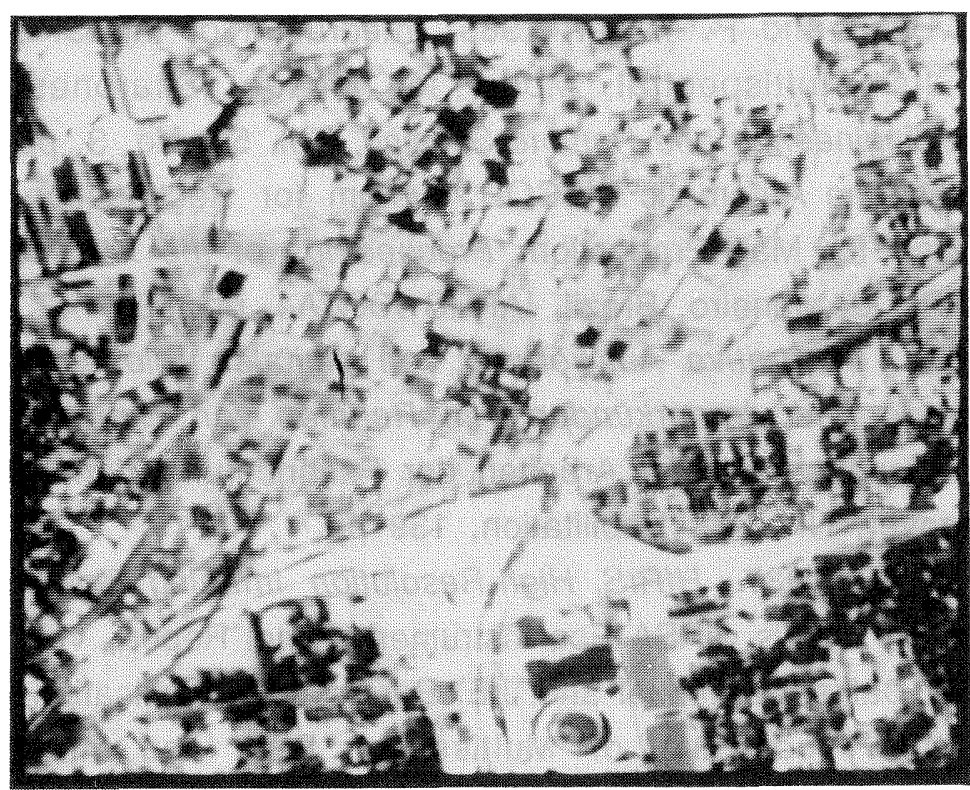

(c)

Figure 10. Black and white prints of (a) a Landsat TM false color image of Atlanta, Georgia resampled to $10-\mathrm{m}$ pixel size; (b) the same TM image after IHS integration with SPOT panchromatic data; and (c) SPOT MSS data integrated with the SPOT panchromatic image. Note the comparable interpretability of (c) and (b).

\section{ACKNOWLEDGMENTS}

SPOT image data are copyrighted (@ 1986) by the Centre National d'Etudes Spatiales, Toulouse, France, and distributed in the U.S. by SPOT Image Corporation, Reston, Virginia.

\section{REFERENCES}

Anderson, R., 1987. Map Information Content of SIR-B Image Data. Master Thesis, The University of Georgia.

Bernstein, R., C. Colby, S.W. Murphrey and J.P. Snyder, 1983. Image Geometry and Rectification. In: R.N. Colwell (Ed.), Manual of Remote Sensing, Second Edition, American Society of Photogrammetry, Vol. I, pp. 873-922.

Butler, D.M. et al., 1986. From Pattern to Process: The Strategy of the Earth Observing System. NASA Eos Steering Committee Report, Vol. II, 140 pp.

Chavez, P.S.Jr., 1986. Digital Merging of Landsat TM and Digitized NHAP data for 1:24,000-scale mapping. Photogrammetric Engineering and Remote Sensing, Vol. 52 , No. 10 , pp. 1637-1646. 
Cliche, G., F. Bonn and P. Teillet, 1985. Integration of the SPOT Panchromatic Channel into its Multispectral Mode for Image Sharpness Enhancement. Photogrammetric Engineering and Remote Sensing, Vol. 51, No. 3, pp. 311-316.

Ehlers, M.; 1984. The Automatic DISCOR System for Rectification of Space-Borne Imagery as a Basis for Map Production. Proceedings of the XVth International Congress of ISPRS. Rio de Janeiro, Brazil, IAPRS XXV/A4, pp. 135-147.

Ehlers, M., 1987. Integrative Auswertung von digitalen Bilddaten aus der Satellitenphotogrammetrie und -fernerkundung im Rahmen von Geographischen Informationssystemen. Wissenschaftliche Arbeiten der Fachrichtung Vermessungswesen der Universität Hannover, Nr. 149, Habilitation, 139 pp.

Goetz, A.F.H. et al., 1987. HIRIS, High-Resolution Imaging Spectrometer: Science Opportunities for the 1990s. NASA Eos Instrument Panel Report, Vol. Ilc, 74 pp.

Haydn, R., G.W. Dalke, J. Henkel and J.E. Bare, 1982. Application of the IHS Color Transform to the Processing of Multisensor Data and Image Enhancement. Proceedings of the International Symposium on Remote Sensing of Arid and SemiArid Lands, Cairo, Egypt, pp. 599-616.

Koger, D.G., 1984. Image Creation for Geologic Analysis and Photointerpretation. Technical Papers, 1984 ASP-ACSM Fall Convention, San Antonio, Texas, pp. 526530.

Luhmann, T. and M. Ehlers, 1984. AlMS: A System for Automatic Image Matching. Proceedings of the Eighteenth International Symposium on Remote Sensing of Environment. Paris, France, pp. 971-979.

Niblack, W., 1986. An Introduction to Digital Image Processing. Prentice-Hall International, $215 \mathrm{pp}$.

Welch, R., 1984. Merging Landsat and SIR-A Image Data in Digital Formats. Imaging Technology in Research and Development, July 1984, pp. 11-12.

Welch, R., T.R. Jordan and M. Ehlers, 1985. Comparative Evaluations of the Geodetic Accuracy and Cartographic Potential of Landsat-4 and Landsat-5 Thematic Mapper Image Data. Photogrammetric Engineering and Remote Sensing, Vol. 51, No. 9, pp. 1249-1262.

Welch, R. and M. Ehlers, 1987. Merging Multiresolution SPOT HRV and Landsat TM Data. Photogrammetric Engineering and Remote Sensing, Vol. 53, No. 3, pp. 301-303. Welch, R. and M. Ehlers, 1988. Cartographic Feature Extraction from Integrated SIR$\mathrm{B}$ and Landsat TM Images. International Journal of Remote Sensing (in press). 